\title{
EFEITO DAS DIMENSÕES DA SEÇÃO TRANSVERSAL E DA FREQUÊNCIA DO TRANSDUTOR NA VELOCIDADE DE PROPAGAÇÃO DE ONDAS DE ULTRA-SOM NA MADEIRA ${ }^{1}$
}

\begin{abstract}
Alex Julio Trinca² e Raquel Gonçalves ${ }^{3}$
RESUMO - Diversas pesquisas vêm sendo realizadas com o uso da velocidade de propagação de ondas de ultra-som como parâmetro para estimar propriedades mecânicas da madeira. Tendo em vista que essa velocidade pode ser influenciada pela forma da peça ensaiada, bem como pela frequência do transdutor utilizado no ensaio, conhecer essas fontes de influência é fator importante para a obtenção de resultados confiáveis. Durante estudo mais amplo sobre a influência da dimensão do corpo-de-prova em ensaios destrutivos de compressão paralela às fibras, os corpos-de-prova de $0,03 \times 0,03 \times 0,09 \mathrm{~m}$ e de $0,05 \times 0,05 \times 0,15$ $\mathrm{m}$ foram também utilizados para avaliar a variação da velocidade de propagação de ondas de ultrassom em razão da dimensão da peça ensaiada. Para a avaliação da influência da frequência, os ensaios foram realizados com transdutores de onda longitudinal (compressão) de $25 \mathrm{kHz}, 45 \mathrm{kHz}, 80 \mathrm{kHz}, 100 \mathrm{kHz}$, $500 \mathrm{kHz}$ e $1 \mathrm{MHz}$, em 119 corpos-de-prova de Pinus elliottii e 244 de Eucalyptus grandis. Os resultados indicaram que, em ambas as espécies, a seção transversal exerceu influência na propagação da onda de ultrassom e que a velocidade longitudinal foi fortemente afetada pela frequência do transdutor para frequências abaixo de $500 \mathrm{kHz}$, corroborando a importância de sempre se adotarem, nos ensaios, relações de comprimento de percurso/comprimento de onda superiores a 3,0 .
\end{abstract}

Palavras-chave: Comprimento de onda, onda longitudinal e onda de compressão.

\section{EFFECT OF THE TRANSVERSAL SECTION DIMENSIONS AND TRANSDUCER FREQUENCY ON ULTRASOUND WAVE PROPAGATION VELOCITY IN WOOD}

\begin{abstract}
Several works use the ultrasound wave propagation velocity as the main parameter to determine the mechanical properties of wood. Since this velocity can be influenced by the specimen's dimension or by the transducer's frequency, this influence must be known to obtain reliable results. During research on the influence of specimen dimension on destructive testing of the compression parallel to the grain, 0.03 $\times 0.03 \times 0.09 \mathrm{~m}$ and $0.05 \times 0.05 \times 0.15 \mathrm{~m}$ specimens were tested to evaluate the variation in wave propagation velocity as a function of specimen dimension and transducer frequency used in the tests. To evaluate transducer influence, the tests were carried out with $25 \mathrm{kHz}, 45 \mathrm{kHz}, 80 \mathrm{kHz}, 100 \mathrm{kHz}, 500 \mathrm{kHz}$ and $1 \mathrm{MHz}$ longitudinal transducers on 119 specimens of Pinus elliottii and 244 specimens of Eucalyptus grandis. The results show that, for both species, the transversal section dimension has an effect on ultrasound wave propagation and that longitudinal velocity was strongly affected by transducer frequency below $500 \mathrm{kHz}$, confirming the importance of adopting during the tests specimen length /wave length above 3.0.
\end{abstract}

Keywords: Wavelength, longitudinal wave and compression wave.

\footnotetext{
${ }^{1}$ Recebido em 21.12.2006 e aceito para publicação em 26.01.2009.

${ }^{2}$ Programa de Pós-Graduação em Engenharia Agrícola da Universidade Estadual de Campinas (UNICAMP). E-mail: <ajtrinca@ terra.com.br>.

${ }^{3}$ Faculdade de Engenharia Agrícola da UNICAMP. E-mail: <raquel@agr.unicamp.br>.
} 


\section{INTRODUÇÃO}

O emprego de técnicas não-destrutivas para avaliar a qualidade e classificar mecanicamente a madeira serrada tem sido foco de muitos estudos e é, certamente, ferramenta imprescindível para a competitividade futura da madeira como material. Entre as técnicas com maiores possibilidades para serem empregadas industrialmente, destacam-se as baseadas em imagens (KHOURY JR., 2005) e em propagação de ondas. No caso de pesquisas com propagação de ondas de ultrassom, a grande maioria vem sendo realizada utilizando, como parâmetro principal de avaliação, a velocidade de propagação.

As equações gerais, apresentadas nos estudos sobre propagação de ondas em meios sólidos, partem do princípio ideal de que o meio de propagação é infinito, ou seja, o comprimento de onda $(\lambda)$ é muito inferior às dimensões da seção transversal do corpo pelo qual essa onda atravessa. Quando a dimensão da peça a ser ensaiada, segundo a direção de propagação da onda ela se aproxima do comprimento de onda $(\lambda)$, o meio passa a se denominar finito e, nesse caso, os fenômenos de reflexão e refração das ondas começam a afetar a propagação e, portanto, a teoria elementar não é mais aplicada diretamente, passando o meio a ser dispersivo.

Além do comprimento da peça, a dimensão da seção transversal (ou do diâmetro) da peça em relação ao diâmetro do transdutor afeta a condição de meio infinito. Quando a dimensão da seção transversal da peça não é, algumas vezes, maior que o diâmetro do transdutor ocorre o fenômeno denominado "efeito de parede". Além disso, se o transdutor envolve toda a seção da peça, o fenômeno da transmissão confina a onda ao corpo-de-prova como uma guia de onda (BUCUR, 2006).

A superposição das ondas produzida pelo pulso (pacote ou trem de ondas) resulta em onda de frequência aproximadamente igual às frequências das ondas originais. Se um pacote de onda mantém a sua forma à medida que se desloca no meio material, todas as ondas harmônicas que o compõem terão a mesma velocidade, mas isso só acontece num meio infinito. No entanto, num meio finito a velocidade de fase (velocidade média das ondas que constituem o trem de ondas) não é igual à velocidade de grupo. Rayleigh (1877) introduziu esse conceito, e Davis (1948) apud Herzig (1992) apresentou curvas experimentais, demonstrando que para $\mathrm{a} / \lambda<$ 0,7 e a $/ \lambda<1,2$ os resultados de velocidade de fase e de grupo, respectivamente, são muito variáveis.
Em trabalho realizado com madeira de "spruce" (Picea abies), a velocidade na direção longitudinal $\left(\mathrm{V}_{\mathrm{LL}}\right)$ foi a mais afetada pela relação entre a largura (b) e a altura (h) da peça. O máximo valor $\mathrm{V}_{\mathrm{LL}}$ foi obtido quando a relação b/h estava entre 1 e 2 , e tanto b quanto $\mathrm{h}$ eram maiores do que o comprimento de onda $(\lambda)$. Em relações b/h acima de 13 a V $_{\text {LL }}$ atingia os valores mínimos e tornava-se praticamente constante (BUCUR, 2006). Essa mesma autora avaliou a variação de $V_{L L}$ quando havia variação tanto de seção quanto de comprimento da peça ensaiada. Nesses ensaios foram utilizados corpos-de-prova de 20 × 20 × $60 \mathrm{~mm}$; 40 x $40 \times 120 \mathrm{~mm}$ e $60 \times 60 \times 180 \mathrm{~mm}$ e transdutores de 1 MHZ de frequência. Ela concluiu que $\mathrm{V}_{\text {LL }}$ variou aproximadamente $12 \%$ entre as peças de comprimentos máximo e mínimo e que essa variação de velocidade não estava relacionada ao comprimento do corpo-deprova, uma vez que a menor relação $1 / \lambda$ foi de 12 , estando, portanto, acima do limite para o qual não deve haver mais influência dessa relação na velocidade. Assim, a variação nesse caso estava relacionada à redução da seção da peça e foi explicada pelo fenômeno do modo de conversão da onda que passa de onda de volume em sólido infinito à onda longitudinal numa "vara".

O campo acústico de um transdutor é subdividido em duas regiões: campo próximo e campo distante. A região de campo próximo caracteriza-se pela ocorrência de interferências construtivas e destrutivas entre as ondas, provocando máximos e mínimos na intensidade do campo acústico. Na região de campo distante, as ondas de ultrassom sofrem somente interferência construtiva, de maneira a formar uma frente de onda quase plana que se atenua à medida que se propaga no meio, distanciando-lhe da fonte. Por ser uma região de interferência, deve-se tomar o cuidado de não ensaiar peças com comprimento inferior ao do campo próximo. O comprimento do campo próximo (N) é função do diâmetro do elemento cristalino (D), da frequência do transdutor (f) e da velocidade de propagação da onda no material (V) e pode ser calculado pela equação $N=\left(D^{2} f\right) /(4$ $\mathrm{V})$ ou $\mathrm{N}=\mathrm{D}^{2} / 4 \lambda$. Além disso, a relação $\mathrm{D} / 1$ indica o padrão de radiação da onda no campo distante.

Em princípio, quanto maior a relação D/1, melhores a resolução e a penetração do sinal. No caso inverso se observa a emissão de componentes indesejáveis de ondas transversais. Entretanto, o comprimento do campo próximo aumenta à medida que a relação $\mathrm{D} / \lambda$ 
se eleva, de forma que é necessário adotar o transdutor que permita melhor adequação do comprimento de campo próximo e do comprimento da peça a ser avaliada.

Para evitar atenuações excessivas, a dimensão do comprimento de onda não deve ser menor do que a dimensão dos elementos anatômicos do material. Na madeira, em frequências entre 1 e 1,5 MHz o comprimento de onda é aproximadamente igual à dimensão de seus elementos anatômicos, de forma que seriam as máximas frequências indicadas para a avaliação desse material (BUCUR, 2006).

Estudos indicam que a velocidade na direção longitudinal $\left(\mathrm{V}_{\mathrm{LL}}\right)$ é a mais afetada pela frequência do transdutor, havendo aumento significativo da velocidade quando se utilizam frequências de $100 \mathrm{kHz}$ a $500 \mathrm{kHz}$. Acima dessa frequência, a velocidade continua aumentando, mas esse aumento é bem menor (Figura 1). Os valores baixos de velocidade (por volta de 3000 $\mathrm{m} / \mathrm{s}$ ) obtidos a $100 \mathrm{kHz}$ foram atribuídos à dispersão geométrica ocorrida devido à relação entre o tamanho do transdutor e a seção da peça (BUCUR, 2006). Esse resultado corrobora os obtidos por Davis (1948) apud Herzig (1992).

Tendo em vista que a frequência do transdutor afeta o comprimento de onda ( $\lambda$ ), é importante destacar alguns trabalhos que não avaliaram diretamente a influência da frequência na velocidade de propagação da onda, mas, indiretamente, por meio do comprimento de onda. Em estudo sobre a variação da velocidade de propagação da onda de ultrassom na madeira de Hêtre (Fagus sylvatica) em razão da relação entre o comprimento do corpo-de-prova (L) e o comprimento de onda $(\lambda)$, ficou demonstrado que a taxa mais acentuada de variação da velocidade longitudinal (30\%) ocorreu entre as relações $L / \lambda=0,1 \mathrm{e} L / \lambda=1$, enquanto nas relações no intervalo $L / \lambda=1 \mathrm{e} L / \lambda=10$ a variação foi de apenas 2,6\% (BUCUR, 2006).

Em outro estudo, tábuas de Eucalyptus grandis com umidade aproximada de $12 \%$, comprimento inicial de $2.000 \mathrm{~mm}$ e seção transversal variando de $50 \times 70$ $\mathrm{mm}$ a $50 \times 140 \mathrm{~mm}$, dois tipos de onda foram avaliados - de superfície e de compressão -, ambas na direção longitudinal. As medições foram feitas com equipamento de ultras-som marca Steinkamp, modelo BP5, com transdutores de $45 \mathrm{kHz}$ de faces planas. Os autores citados concluíram que para a relação entre comprimento de percurso da onda (d) e comprimento de onda $(\lambda)$ maior do que 5 as ondas de superfície se transformam em ondas de compressão com mesma velocidade de propagação, além de representarem intervalo em que a velocidade de propagação da onda deixa de ser afetada pelo comprimento de percurso. Assim, para relações $\mathrm{d} / \lambda$ acima desse valor, tanto ondas de superfície quanto de compressão podem ser utilizadas para avaliação do material. Além disso, os referidos autores concluíram que é recomendado que a seção transversal tenha relação $\mathrm{R} / \mathrm{T}$ maior do que 0,3 para que a velocidade na direção longitudinal não seja afetada e R/T maior do que 0,75 para que a velocidade na direção tangencial também não seja afetada (BARTHOLOMEU et al., 2003).

Outro estudo sobre a variação da velocidade ultrassônica longitudinal em função do comprimento da peça de madeira utilizando transdutor de frequência $22 \mathrm{kHz}$ foi conduzido utilizando-se as espécies: Pinus caribaea var. caribaea, Eucalyptus citriodora, Eucalyptus grandis e Hymenaea sp (jatobá). Foram feitas medições do tempo de propagação das ondas ultrassônicas, com o comprimento das peças sendo reduzido de 3.000 a $100 \mathrm{~mm}$ e mantendo-se a seção transversal constante de $120 \times 50 \mathrm{~mm}$. Os resultados indicaram que existe ponto crítico na relação comprimento de percurso (L) e comprimento de onda $(\lambda)$, a partir do qual não ocorre mais interferência na velocidade obtida e é necessário que o corpo-de-prova tenha comprimento superior a três vezes o comprimento de onda (GOIA et al., 2006).

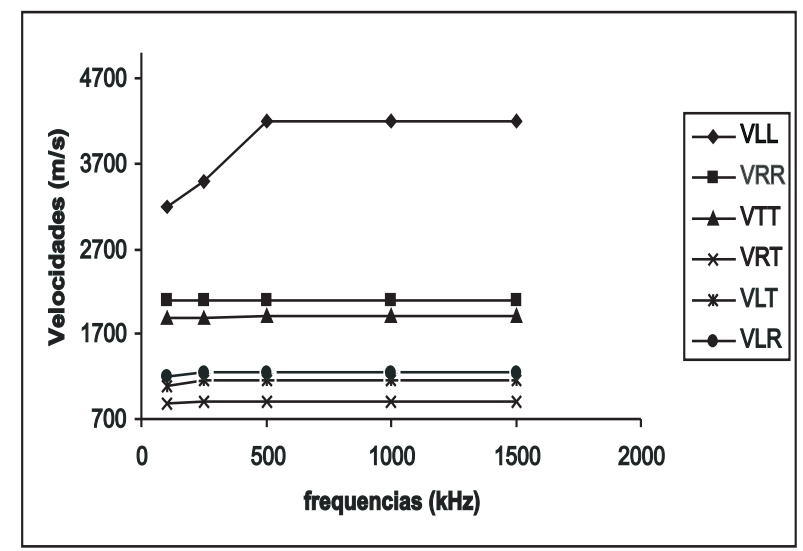

Fonte: adaptado de BUCUR, 2006. Source: BUCUR, 2006.

Figura 1 - Influência da frequência na medição da velocidade. Figure 1 - Influence of frequency on ultrasonic velocity measurements.

R. Árvore, Viçosa-MG, v.33, n.1, p.177-184, 2009 
O objetivo deste trabalho foi avaliar a influência da frequência do transdutor e da dimensão da seção transversal do corpo-de-prova na velocidade de propagação longitudinal de ondas de ultrassom na madeira de Pinus elliottii (conífera) e de Eucalyptus grandis (dicotiledônea).

\section{MATERIAL E MÉTODOS}

Nos ensaios foram utilizados 119 corpos-de-prova de Pinus elliottii ( 83 com seção transversal de $30 \mathrm{~mm}$ (R) $\times 30 \mathrm{~mm}$ (T) e $90 \mathrm{~mm}$ de comprimento (L) e $36 \mathrm{com}$ seção transversal $50 \mathrm{~mm}(\mathrm{R}) \times 50 \mathrm{~mm}(\mathrm{~T})$ e comprimento (L) de $150 \mathrm{~mm}$ ) e 244 corpos-de-prova de Eucalyptus grandis (159 com seção transversal de $30 \mathrm{~mm}(\mathrm{R}) \times$ $30 \mathrm{~mm}$ (T) e $90 \mathrm{~mm}$ de comprimento (L) e 85 com seção transversal de $50 \mathrm{~mm}(\mathrm{R}) \times 50 \mathrm{~mm}(\mathrm{~T})$ e comprimento (L) de $150 \mathrm{~mm}$ ).

Tendo em vista que os ensaios não-destrutivos foram realizados no escopo de um projeto mais amplo que visava, também, à avaliação da influência do tamanho do corpode-prova no ensaio destrutivo de compressão paralela às fibras, a metodologia utilizada foi diferente da usual para esse tipo de avaliação. Normalmente para se determinar a influência das dimensões da peça na velocidade de propagação das ondas de ultrassom os ensaios são realizados em uma mesma peça, cujo comprimento e seção transversal vão sendo reduzidos. No caso deste trabalho, no entanto, os ensaios tiveram que ser realizados em corpos-de-prova distintos, uma vez que seriam rompidos. Para minimizar as influências da variabilidade do material, a retirada dos corpos-de-prova foi realizada, intercalandose a posição dos corpos-de-prova de seções distintas na peça de origem (Figura 2). Tal procedimento garantiu que a variabilidade fosse distribuída entre as peças de diferentes seções, não concentrando alguma característica em peças de mesma seção.

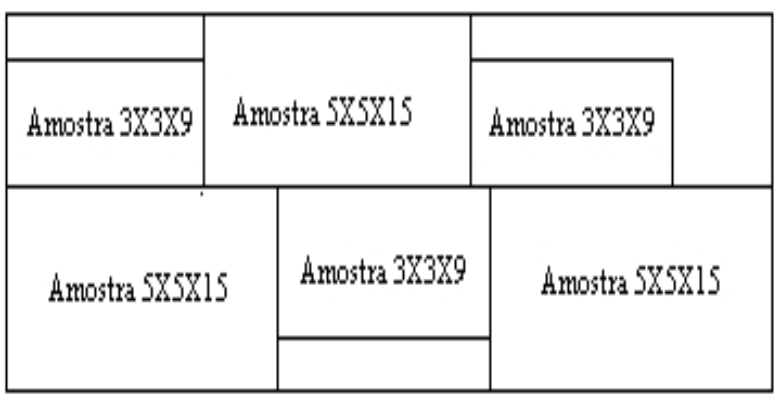

Figura 2 - Esquema de amostragem dos corpos-de-prova. Figure 2-Sampling scheme for the specimens.

R. Árvore, Viçosa-MG, v.33, n.1, p.177-184, 2009
Os corpos-de-prova foram previamente climatizados em câmara climática, com controle de temperatura e de umidade relativa até atingirem umidade de equilíbrio de aproximadamente $12 \%$. Além disso, o teor de umidade foi medido imediatamente antes do ensaio, fazendose uso de medidor de umidade superficial de três polos, o qual foi previamente aferido. Para a aferição do medidor de umidade foi elaborada a curva de calibração, utilizando-se o ensaio de determinação de umidade da norma NBR 7190/97: Projeto de Estruturas de Madeira. O resultado dessa calibração indicou desvio-padrão de $+/-0,3 \%$ na faixa de trabalho desejada.

A densidade aparente dos corpos-de-prova, na umidade de equilíbrio, foi determinada utilizando-se as especificações da NBR 7190/97: Projeto de Estruturas de Madeira.

Nos ensaios de ultrassom, utilizou-se equipamento EPOCH4 da Panametrics com transdutores de onda longitudinal e faces planas de $25 \mathrm{kHz}, 45 \mathrm{kHz}, 80 \mathrm{kHz}$, $100 \mathrm{kHz}, 500 \mathrm{kHz}$ e $1.000 \mathrm{kHz}$ de frequência. Cada corpode-prova foi ensaiado com transdutores de todas as frequências indicadas, totalizando 1.464 medições no Eucalyptus grandis e 714 medições no Pinus elliottii.

Com o ensaio de ultrassom, determinou-se o tempo de propagação da onda na direção longitudinal $\left(\mathrm{t}_{\mathrm{LL}}\right)$. Os ensaios foram realizados no Laboratório Físico da Madeira, da Escola SENAI de Itatiba. Para determinação da velocidade de propagação da onda na direção longitudinal $\left(\mathrm{V}_{\mathrm{LL}}\right)$, os transdutores foram posicionados nas faces do corpo-de-prova, de maneira que a propagação ocorresse na direção longitudinal com onda de compressão. Os ensaios foram sempre realizados com o uso de acoplante entre o transdutor e o corpode-prova. O acoplante utilizado foi gel medicinal. De posse do tempo de propagação da onda $\left(t_{L L}\right)$ e do comprimento de percurso ( $\mathrm{L}=$ comprimento do corpode-prova), determinou-se a velocidade de propagação da onda. As determinações dos comprimentos de onda foram feitas utilizando-se a velocidade média (considerando-se as duas seções de corpos-de-prova) obtida de cada espécie e em cada frequência de transdutor empregada no ensaio.

\section{RESULTADOS E DISCUSSÕES}

Na Tabela 1, apresentam-se os valores médios das umidades e densidades aparentes referentes aos corpos- 
de-prova de Eucalyptus grandis e de Pinus elliottii. Verifica-se, nesta tabela, que a umidade média dos corposde-prova se manteve próxima da umidade de referência (12\%), com variabilidades pequenas (em torno de 5\%). A densidade aparente média dos corpos-de-prova de Pinus elliottii foi próxima da densidade média tabelada na NBR 7190/97: Projeto de Estruturas de Madeira, que é de $560 \mathrm{~kg} \cdot \mathrm{m}^{-3}$. Já no Eucalyptus grandis a densidade aparente média dos corpos-de-prova foi $36 \%$ menor do que a indicada na norma citada, confirmando tratarse de madeira juvenil. Essa constatação já havia sido verificada em outros ensaios realizados com o mesmo lote da madeira.

Na Tabela 2, apresentam-se dados relativos aos transdutores utilizados (diâmetro do elemento (D), o diâmetro do transdutor (T)) e os valores do campo próximo $(\mathrm{N})$ e da relação $\mathrm{D} / \lambda$. O campo próximo foi calculado de acordo com a equação apresentada no item 1 .

Verificou-se que os transdutores de frequência acima de $500 \mathrm{kHz}$ apresentaram relação D/1 em torno de 2 , indicando os mesmos índices de penetração e resolução. Esses índices também evidenciaram que esses transdutores possuíam maior grau de penetração e melhor resolução quando comparados com os transdutores de menor frequência, uma vez que a relação $D / \lambda$ foram superiores. Nas duas seções ensaiadas, o comprimento dos corpos-de-prova (90 mm e $150 \mathrm{~mm}$ ) foi bem maior do que o comprimento do campo próximo, evitando-se medições afetadas nesse trecho.

Em termos da relação entre a dimensão do transdutor e a dimensão da seção transversal da peça, verificouse que, nos corpos-de-prova de seção $30 \times 30 \mathrm{~mm}$, somente o transdutor de $1 \mathrm{MHz}$ atendia à condição de estar inserido na seção, pois seu diâmetro é de 18 $\mathrm{mm}$. Em todos os demais haverá o problema da guia de onda relatado por Bucur (2006). O transdutor de $500 \mathrm{kHz}$, embora não atenda totalmente a essa condição para essa dimensão de seção transversal, também será considerado na avaliação, uma vez que pelo menos o elemento se encontra circunscrito à seção transversal. Para o caso da seção $50 \times 50 \mathrm{~mm}$, todos os transdutores estão circunscritos à seção. Adicionalmente, em ambas as seções, nos transdutores de frequência abaixo de $500 \mathrm{kHz}$ a relação $\mathrm{a} / \lambda$ (considerando $\mathrm{a}=$ metade da largura da seção transversal) estariam abaixo de 1,2, o que de acordo com Davis (1048) apud Herzig (1992) implicaria velocidades de fase e de grupo diferenciadas e, portanto, a propagação não estaria se dando em condições de meio infinito.

Tabela 1 - Valores médios das umidades e das densidades aparentes obtidas dos corpos-de-prova de Eucalyptus grandis e de Pinus elliottii. Os valores entre parênteses correspondem ao coeficiente de variação

Table 1 -Average for moisture content and density for specimens of Eucalyptus and Pinus elliottii. The values on parentheses correspond to the variation coefficient

\begin{tabular}{|c|c|c|c|c|}
\hline & $\begin{array}{c}\text { Eucalyptus grandis } \\
(3 \times 3 \times 9)\end{array}$ & $\begin{array}{l}\text { Eucalyptus grandis } \\
\quad(5 \times 5 \times 15)\end{array}$ & $\begin{array}{l}\text { Pinus elliottii } \\
(3 \times 3 \times 9)\end{array}$ & $\begin{array}{l}\text { Pinus elliottii } \\
(5 \times 5 \times 15)\end{array}$ \\
\hline Umidade & 11,6 & 12,2 & 11,6 & 11,5 \\
\hline$(\%)$ & $(5,5 \%)$ & $(5,4 \%)$ & $(6,2 \%)$ & $(5,7 \%)$ \\
\hline Densidade & 467 & 472 & 565 & 564 \\
\hline$\left(\mathrm{kg} / \mathrm{m}^{3}\right)$ & $(7,9 \%)$ & $(6,5 \%)$ & $(14,6 \%)$ & $(11,2 \%)$ \\
\hline
\end{tabular}

Tabela 2 - Dados dos transdutores utilizados nos ensaios

Table 2 - Transducer data used during the tests

\begin{tabular}{|c|c|c|c|c|}
\hline \multirow[t]{2}{*}{ Frequência $(\mathrm{kHz})$} & \multicolumn{2}{|c|}{ Diâmetros $(\mathrm{mm})$} & \multicolumn{2}{|c|}{ Campo próximo $(\mathrm{N})(\mathrm{mm})$} \\
\hline & Elemento (D) & Transdutor $(\mathrm{T})$ & Eucalitpto & Pinus elliottii \\
\hline 25 & 25 & 32 & $0,78(0,12)$ & $0,74(0,12)$ \\
\hline 45 & 25 & 32 & $1,42(0,23)$ & $1,40(0,22)$ \\
\hline 80 & 25 & 32 & $2,26(0,36)$ & $2,14(0,34)$ \\
\hline 100 & 32 & 44 & $4,65(0,6)$ & $4,57(0,6)$ \\
\hline 500 & 25 & 32 & $13,0(2,1)$ & $13,0(2,1)$ \\
\hline 1000 & 13 & 18 & $7,0(2,2)$ & $6,8(2,1)$ \\
\hline
\end{tabular}

* O valor entre parênteses indica o valor aproximado da relação $\mathrm{D} / \lambda$. 
Na Tabela 3, apresentam-se, no caso de Eucalyptus grandis e Pinus elliottii, valores dos comprimentos de onda $(\lambda)$, bem como as relações entre comprimento de percurso (L) e comprimento de onda $(\lambda)$, obtidos utilizando-se os transdutores de diferentes frequências e os diferentes comprimentos de percurso. Observouse que, nas dimensões das peças ensaiadas, somente com os transdutores de frequência acima de $500 \mathrm{kHz}$ foi possível obter-se relação $L / \lambda$ superior a 3,0 . Com base nas conclusões obtidas por Bartholomeu et al. (2003) e Goia (2006), somente no caso desses transdutores os valores de velocidade não estariam afetados pela relação $L / \lambda$, sendo, portanto, os mais indicados para as referidas seções avaliadas. Além da questão da relação $\mathrm{L} / \lambda$, verificou-se que somente para os transdutores de frequência acima de $500 \mathrm{kHz}$ a seção transversal da peça ensaiada foi maior do que o comprimento de onda. Dessa forma, de acordo com Bucur (2006) e Herzig (1992), os valores de velocidade obtidos nas demais frequências estariam afetados pelo fenômeno da conversão de onda.

Na Tabela 4 são apresentados os valores médios de velocidade obtidos nas diferentes frequências e nas duas espécies estudadas. Em todas as frequências analisadas, observou-se que os coeficientes de variação da velocidade foram bem maiores em Pinus elliottii (média de 14,1\%) do que em Eucalyptus grandis (média de 5,6\%). Esse resultado está relacionado à maior homogeneidade das peças de Eucalyptus grandis, uma vez que, nas peças de Pinus elliottii, havia maior incidência de nós e desvio de fibras. Verificou-se também que no corpo-de-prova de seção $50 \times 50 \mathrm{~mm}$ a variabilidade foi levemente menor do que no de seção $30 \times 30 \mathrm{~mm}$, em ambas as espécies. Resultado esse que possivelmente está relacionado às questões relativas ao tamanho da peça em relação ao diâmetro do transdutor e ao comprimento de onda, fatores esses que afetam as medições (BUCUR, 2006).

Tendo em vista as discussões relativas às interferências dos aspectos comprimento de onda e diâmetro do transdutor na velocidade de propagação obtida nos ensaios, a avaliação da influência da dimensão do corpo-de-prova em $\mathrm{V}_{\mathrm{LL}}$ foi restrita aos resultados obtidos com os transdutores de $500 \mathrm{kHz}$ e $1.000 \mathrm{kHz}$. Nessas frequências, as condições do ensaio foram mais próximas das condições de contorno da teoria de meios infinitos.

Tabela 3 - Valores de comprimento de onda (1) e de relação entre comprimento de percurso (L) e comprimento de onda (1) nos transdutores de diferentes frequências

Table 3 - Values of wave length $(l)$ and route length $(L)$ and wave length $(l)$ ratio for transducers of different frequencies

\begin{tabular}{|c|c|c|c|c|c|c|}
\hline \multirow{3}{*}{ Frequência $(\mathrm{kHz})$} & \multicolumn{3}{|c|}{ Eucalyptus grandis } & \multicolumn{3}{|c|}{ Pinus elliottii } \\
\hline & \multirow[t]{2}{*}{$\lambda(\mathrm{mm})$} & \multicolumn{2}{|c|}{$\mathrm{L} / \lambda$} & \multirow{2}{*}{$\overline{\lambda(\mathrm{mm})}$} & \multicolumn{2}{|c|}{$L / \lambda$} \\
\hline & & $\mathrm{L}=90 \mathrm{~mm}$ & $\mathrm{~L}=150 \mathrm{~mm}$ & & $\mathrm{~L}=90 \mathrm{~mm}$ & $\mathrm{~L}=150 \mathrm{~mm}$ \\
\hline 25 & 200 & 0,45 & 0,75 & 210 & 0,43 & 0,71 \\
\hline 45 & 110 & 0,82 & 1,36 & 112 & 0,80 & 1,34 \\
\hline 80 & 69 & 1,30 & 2,17 & 73 & 1,23 & 2,05 \\
\hline 100 & 55 & 1,64 & 2,73 & 56 & 1,61 & 2,68 \\
\hline 500 & 12 & 7,5 & 12,5 & 12 & 7,5 & 12,5 \\
\hline 1000 & 6 & 15 & 25 & 6,2 & 14,5 & 24,2 \\
\hline
\end{tabular}

Tabela 4 - Velocidades médias das duas espécies obtidas nas diferentes frequências estudadas. Os valores entre parêntesis indicam o coeficiente de variação dos resultados

Table 4 - Mean velocities for both species obtained on the different frequencies studied. The parenthesis values indicate the variation coefficient of the results

\begin{tabular}{|c|c|c|c|c|}
\hline \multirow[t]{3}{*}{ Frequência $(\mathrm{kHz})$} & \multicolumn{4}{|c|}{ Velocidade } \\
\hline & \multicolumn{2}{|c|}{ Eucalyptus grandis } & \multicolumn{2}{|c|}{ Pinus elliottii } \\
\hline & $(3 \times 3 \times 9)$ & $(5 \times 5 \times 15)$ & $(3 \times 3 \times 9)$ & $(5 \times 5 \times 15)$ \\
\hline 25 & $4964(6,0 \%)$ & $4998(4,0 \%)$ & $5249(13,4 \%)$ & $5203(12,4 \%)$ \\
\hline 45 & $4934(6,2 \%)$ & $5005(3,8 \%)$ & $4993(13,9 \%)$ & $5107(12,5 \%)$ \\
\hline 80 & $5741(6,3 \%)$ & $5306(4,8 \%)$ & $5968(14,6 \%)$ & $5625(12,8 \%)$ \\
\hline 100 & $5571(6,2 \%)$ & $5500(5,1 \%)$ & $5780(13,8 \%)$ & $5477(15,0 \%)$ \\
\hline 500 & $6108(6,0 \%)$ & $5731(5,4 \%)$ & $6703(14,4 \%)$ & $5850(15,0 \%)$ \\
\hline 1000 & $6279(6,9 \%)$ & $5743(5,6 \%)$ & $6620(16,2 \%)$ & $5859(15,0 \%)$ \\
\hline Geral* & 5600 & 5381 & 5886 & 5458 \\
\hline
\end{tabular}

*Media de velocidades obtidas com as diferentes frequências ensaiadas.

R. Árvore, Viçosa-MG, v.33, n.1, p.177-184, 2009 
$\mathrm{Na}$ Tabela 5, apresenta-se o resultado do teste de comparação de médias das velocidades obtidas nos corpos-de-prova de $30 \times 30 \mathrm{~mm}$ e $50 \times 50 \mathrm{~mm}$, com transdutores de frequência de $500 \mathrm{kHz}$ e de 1.000 $\mathrm{kHz}$ nas duas espécies ensaiadas. O teste estatístico foi efetuado utilizando-se o Programa Statgraphics Plus. Tendo em vista que em todos os casos analisados o intervalo de diferença de médias não contém o zero, pode-se concluir que as velocidades são estatisticamente diferentes, com $95 \%$ de nível de confiança. Tendo em vista que $\mathrm{L} / \mathrm{l}$ nas duas dimensões de corpos-de-prova estavam acima de 7,0, pode-se concluir que a variação na velocidade foi ocasionada unicamente pela variação na dimensão da seção transversal. As diferenças médias de velocidade, considerando-se as duas frequências analisadas, foram de $8 \%$ em Eucalyptus grandis e de 13,5\% em Pinus elliottii. Resultado próximo (diferença média de $12 \%$ ) foi obtido por Bucur (2006).

As Figuras 3 e 4 ilustram o comportamento de $\mathrm{V}_{\mathrm{LL}}$ em função da frequência do transdutor no Eucalyptus grandis e Pinus elliottii, respectivamente, nas duas seções de peça ensaiadas.

Verifica-se, na Figura 1, nas duas espécies, comportamento semelhante aos obtidos por Bucur (2006) ou seja, a velocidade se eleva com o aumento da frequência do transdutor, sendo esse aumento mais acentuado nas frequências de até $500 \mathrm{kHz}$; acima dessa frequência, a velocidade se mantém praticamente constante.

Observando o gráfico da Figura 1, verifica-se que a inclinação da reta (relação da variação da velocidade pela variação da frequência) é de aproximadamente 2,0 no trecho correspondente a 100 e $500 \mathrm{kHz}$. No caso dos resultados desta pesquisa, a inclinação foi em torno de 2,0 nos corpos-de-prova de seção $30 \times 30 \mathrm{~mm}$ e em torno de 1,0 nos corposde-prova de $50 \times 50 \mathrm{~mm}$, considerando-se o trecho correspondente a 25 e $500 \mathrm{kHz}$. Em ambas as espécies e seções, a partir de $500 \mathrm{kHz}$ a variação da velocidade (dada pelo coeficiente angular da reta obtida na regressão) apresentou significativa redução, passando a aproximadamente 0,3 nos corpos-de-prova de 30 $\times 30 \mathrm{~mm}$ e cerca de 0,1 nos corpos-de-prova de 50 $\times 50 \mathrm{~mm}$.
Tabela 5 - Intervalo de confiança (95\%) da diferença entre médias de $\mathrm{V}_{\mathrm{LL}}$ obtidas em corpos- -de-prova de $303090 \mathrm{~mm}$ e $5050150 \mathrm{~mm}$

Table 5 - Confidence interval (95\%) for the difference between the means of the VLL obtained in specimens of the $303090 \mathrm{~mm}$ and $5050150 \mathrm{~mm}$

\begin{tabular}{lcc}
\hline & $500 \mathrm{KHz}$ & $1.000 \mathrm{~K} \mathrm{~Hz}$ \\
\hline Espécies & Intervalo de confiança \\
& da diferença entre médias de $\mathrm{V}_{\mathrm{LL}}(\mathrm{m} / \mathrm{s})$ \\
\hline Eucalyptus grandis & $284-469$ & $429-641$ \\
Pinus elliottii & $859-1591$ & $358-1164$ \\
\hline
\end{tabular}

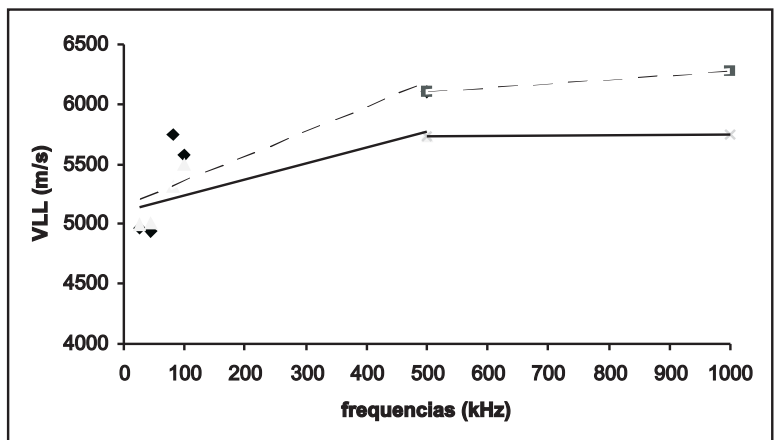

A linha pontilhada representa os resultados dos corpos-de-prova de 30 x $30 \mathrm{~mm}$; e a linha contínua, os resultados dos corpos-de-prova de $50 \times 50 \mathrm{~mm}$.

Figura 3 - Influência da frequência na medição da velocidade de propagação de ondas de ultrassom. Espécie: Eucalyptus grandis.

Figure 3 - Influence of frequency on ultrasonic velocity measurements. Species: Eucalyptus grandis.

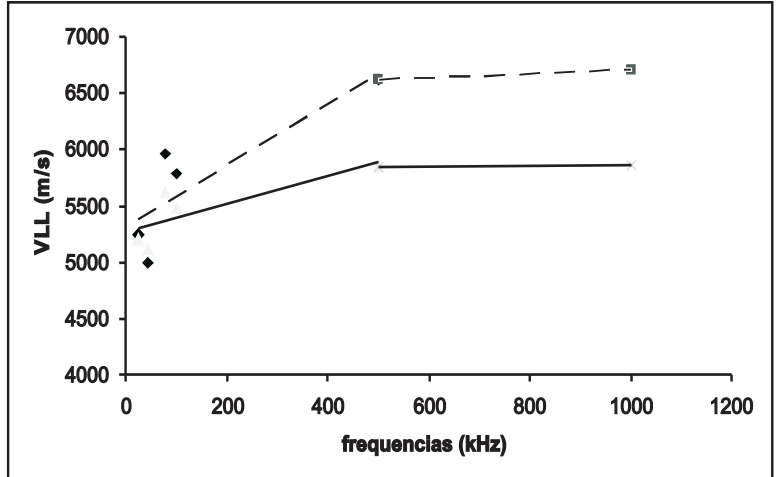

A linha pontilhada representa os resultados dos corpos-de-prova de 30 x $30 \mathrm{~mm}$; e a linha contínua, os resultados dos corpos-de-prova de $50 \times 50 \mathrm{~mm}$.

Figura 4 - Influência da frequência na medição da velocidade de propagação de ondas de ultrassom. Espécie: Pinus elliottii.

Figure 4 - Influence of frequency on ultrasonic velocity measurements. Species: Pinus elliottii.

R. Árvore, Viçosa-MG, v.33, n.1, p.177-184, 2009 
Os resultados de velocidade nas duas diferentes seções indicaram maiores diferenças em Pinus elliottii do que em Eucalyptus grandis. Em ambas as espécies, no entanto as diferenças de velocidade entre as seções foram muito pequenas para frequências de até $100 \mathrm{kHz}$, sendo significativas estatisticamente somente a partir de $500 \mathrm{kHz}$. A explicação para esse comportamento são as interferências no ensaio devido a efeitos ocasionados pela execução do ensaio fora das condições ideais para a propagação da onda (relação $L / \lambda<3,0$; $\mathrm{b}$ e $\mathrm{h}<\lambda ; \mathrm{a} / \lambda<1,2$; dimensão do transdutor maior do que a dimensão da peça), que mascaram as diferenças reais obtidas quando as condições são mais próximas das teóricas.

Finalmente, é importante destacar que, levandose em consideração dados referentes à anatomia da madeira de Eucalyptus grandis, obtidos por Brasil e Ferreira (1972) em peças retiradas de povoamentos de 16 anos; e de Pinus elliottii, obtidos por Foelkel et al. (1975) em peças retiradas de povoamentos de 14 anos, ambos no Estado de São Paulo, o comprimento de onda para o transdutor de $1 \mathrm{MHz}$ foi próximo do comprimento de vaso no Eucalyptus grandis $(5 \mathrm{~mm})$ e de traqueide no Pinus elliottii (4 mm), sendo, portanto, a frequência-limite para a execução de ensaios nas dimensões utilizadas neste trabalho.

\section{CONCLUSÕES}

Após a avaliação e discussão dos resultados são apresentadas as seguintes conclusões:

- Nas frequências do transdutor maiores que 500 $\mathrm{kHz}$, as velocidades de propagação das ondas na direção longitudinal $\left(\mathrm{V}_{\mathrm{LL}}\right)$ foram estatisticamente diferentes nas duas seções transversais estudadas. Esse resultado indica que a seção transversal exerce influência na propagação da onda de ultrassom, tendo sido da ordem de $8 \%$ no eucalipto e $13 \%$ no Pinus elliottii.

- Nas frequências abaixo de $500 \mathrm{kHz}$, as diferenças de velocidade entre as duas diferentes seções não foram estatisticamente significativas, provavelmente devido às interferências discutidas no item anterior, que não permitiram que as diferenças reais, obtidas quando as condições são mais próximas das teóricas, fossem percebidas.

- A velocidade $\mathrm{V}_{\mathrm{LL}}$ foi fortemente afetada pela frequência do transdutor para frequências abaixo de
$500 \mathrm{kHz}$, corroborando a importância de sempre se adotarem nos ensaios relações comprimento de percurso/ comprimento de onda superiores a 3,0. Quando essa relação não é obedecida, os valores de velocidade de propagação das ondas obtidos podem estar afetados, podendo ser utilizados em análises comparativas, mas não em determinações da matriz de rigidez.

- Aspectos relacionados ao transdutor, como o campo próximo, o diâmetro e a frequência, devem ser sempre analisados para que a sua escolha seja mesmo compatível com as dimensões e estrutura anatômica do material a ser ensaiado.

\section{REFERÊNCIAS}

\section{ASSOCIACÃO BRASILEIRA DE NORMAS} TÉCNICA. NBR 7190/97: Projeto de estruturas de madeira. Rio de Janeiro: 1997. 107p.

BARTHOLOMEU, A.; GONÇALVES, R.; BUCUR V. Dispersion of ultrasonic waves in Eucalyptus lumber as a function of the geometry of boards. Scientia Forestalis, n.63, p.235-240, 2003.

BUCUR, V.. Acoustics of wood. 2.ed. New York: Springer-Verlag, 2006. 393p.

HERZIG, L. Evaluation du module dÝoung de bois d'épinettepar méthode ultrasonore sur carottes de sondage. 1992. 290f. Tese (Doutorado - Génie Forestière) Université Laval, Québec, 1992.

GOIA, F. R. O. et al. Efeito do comprimento do corpode-prova na velocidade ultra-sônica em madeiras. Revista Árvore, v.30, n.1, p.141-145, 2006.

FOELKEL, C. E. B. et al. Variabilidade no sentido radial de madeira de Pinus elliottii elliottii.

Scientia Forestalis, n.10, p.1-11, 1975.

BRASIL, M. A. M.; FERREIRA, M. Variação da densidade básica e das características das fibras em Eucalyptus grandis HILL EX MAIDEN ao nível do DAP. Scientia Forestalis, n.5, p.81-90, 1972.

KHOURY JUNIOR, J. K. et al.. Análise discriminante paramétrica para reconhecimento de defeitos em tábuas de eucalipto utilizando imagens digitais. Revista Árvore, v.29, n.2, p.299-309, 2005. 